\title{
Coexistence of quasi-CW and SBS-boosted self- Q-switched pulsing in ytterbium-doped fiber laser with low $Q$-factor cavity
}

\author{
Yuri O. Barmenkov, Member, IEEE, Pablo Muniz-Cánovas, Alexander V. Kir'yanov, Member, IEEE, \\ Antonio A. Carrascosa, Jose L. Cruz, and Miguel V. Andrés, Member, IEEE
}

\begin{abstract}
We report the results of an experimental study of an ytterbium-doped fiber laser with low Q-factor cavity. We demonstrate that the laser operates in two randomly alternating sub-regimes, quasi-CW (QCW) and self-Q-switching (SQS), the latter ignited by stimulated Brillouin scattering (SBS). We show that probability of each sub-regime depends on pump power: QCW dominates slightly above the laser threshold while SQS pulsing prevails at higher pump powers. We also discuss the featuring details of QCW sub-regime and its role in instabilities (jittering) of SBS-boosted SQS pulsing as well as the statistical properties of the latter.
\end{abstract}

Index Terms-ytterbium-doped fiber laser, stimulated Brillouin scattering, self-Q-switching, supercontinuum.

\section{INTRODUCTION}

Y tterbium-doped fiber lasers (YDFLs) are efficient devices with optical efficiency as high as $\sim 80 \%$ [1]-[3], limited mostly by Stokes-shift loss and, also, by the concentration [4],[5] and photodarkening [5]-[10] phenomena inherent in ytterbium-doped fiber (YDF). Output power of YDFLs reaches multi-kW [1]-[3],[11],[12] and even few-hundred kW [12]-[14] levels in single-mode and multi-mode fiber geometries, respectively. Thus, because of the excellent power budget and very large power scaling, this type of fiber lasers (FLs) finds a large number of technological applications.

When Q-factor of the cavity is high such lasers usually operate in $\mathrm{CW}$ (or quasi-CW), characterized by excessive

Manuscript received August 28, 2019. This work was supported in part by the Agencia Estatal de Investigación (AEI) of Spain and Fondo Europeo de Desarrollo Regional (FEDER) (Ref. TEC2016-76664-C2-1-R). The work of P. Muniz-Cánovas was supported by the CONACyT Doctoral Fellowship Program (Mexico). The work of Y. O. Barmenkov was supported by the CONACyT Sabbatical Program. The work of A. V. Kir'yanov was supported in part by the Increase Competitiveness Program of NUST "MISIS" of the Ministry of Education and Science (Russian Federation) under Grant K32018-23. (Corresponding author: Yuri O. Barmenkov).

P. Muniz-Cánovas, Y. O. Barmenkov, and A. V. Kir'yanov are with the Centro de Investigaciones en Óptica A.C. (e-mails: pablomc@cio.mx; yuri@cio.mx; kiryanov@cio.mx).

A. V. Kir'yanov is with the National University of Science and Technology "MISIS", Moscow 119049, Russian Federation.

Yuri O. Barmenkov, A. A. Carrascosa, J. L. Cruz, and M. V. Andrés are with the Departamento de Fisica Aplicada, Instituto de Ciencia de Materiales, Universidad de Valencia, 46100 Valencia, Spain (e-mails: yuri@cio.mx, antonio.carrascosa@uv.es, jose.1.cruz@uv.es; miguel.andres@uv.es). photon noise [15],[16] described by Bose-Einstein statistics [15],[17]. However, YDFLs with low Q-factor cavities turn to $\mathrm{kW}$-level random self-Q-switching (SQS), ignited by stimulated Brillouin scattering (SBS) [18]-[22]. In our previous work concerning YDFL operation in the regime of SBS pulses [20], we showed that stability of such kind of pulses strongly improves with increasing intra-cavity loss to the level above which the laser operates as a source of broadband amplified spontaneous emission. Recently SBSbased SQS YDFLs were proposed as a simple pump source of nanosecond pulses for broadband supercontinuum (SC) generation [20],[23],[24] and, also, as rogue waves' oscillators [22]. Certainly, such lasers present a more robust and cheaper alternative to generate SC as compared picosecond (modelocked) tandem YDF-based systems [25],[26].

However, SBS-SQS pulses (thereafter "SBS pulses") are characterized by strong amplitude and timing jitter resulted from the stochastic nature of SBS process, which is a strong drawback for most applications. Some efforts were made to resolve the problem of jitter. Say, it was shown that jittering of SBS-pulses fades with increasing intra-cavity loss [20]. Also, it was proposed to use a pulsed distributed feed-back (DFB) laser as source of stable pulsed seed signal for igniting SBS pulses [27]; in this case, SBS-boosting of the seed pulses occurs in a multi-pass amplifying regime; this allows jitter mitigating but not its elimination. However, the inclusion of additional expensive elements into the scheme leads to the loose of simplicity and increases the cost of such lasers but does not fully resolve the problem.

In this work, we show that an YDFL with very low cavity Q-factor operates in two coexisting (each other alternating) sub-regimes: quasi-CW (QCW) and SBS pulsing. Probability of each sub-regime depends on pump level: at low pump powers, probability of QCW pulsing is much higher than the SBS one and, vice versa, at high pump powers SBS (SQS) pulsing dominates. We also discuss some featuring characteristics of the laser in each sub-regime and the effect of intermittent QCW windows as a source of instabilities (jittering) of SBS pulses. The statistical properties of SBS pulsing, addressed in terms of amplitude and timing jitters, are studied in detail, too. 


\section{EXPERIMENTAL SETUP AND BASIC LASER PARAMETERS}

Figure 1(a) shows a layout of the YDFL with Fabry-Perot cavity. YDF used was a standard double-clad (DC) fiber with cladding absorption $\sim 1.65 \mathrm{~dB} / \mathrm{m}$ at $975 \mathrm{~nm}$, numerical aperture $N A=0.12$, and mode field diameter $M F D \approx 6.5 \mu \mathrm{m}$ at the laser wavelength $(1061.5 \mathrm{~nm})$; YDF length was $8 \mathrm{~m}$. The laser was pumped by a commercial 975 -nm laser diode (LD) through a pump combiner.

The laser cavity was formed by in-house made uniform fiber Bragg grating (FBG) with narrow reflection spectrum (see discussion below) placed at its left side and by very weak reflection from isolator ISO1 (return loss is $50 \mathrm{~dB}$ ) placed at its right side; the total cavity length, $L_{c}$, was $12.7 \mathrm{~m}$. The FBG was managed to have relatively low reflection $(\approx 10 \%$ at $1061.5 \mathrm{~nm}$ ) that, in combination with weak feedback on the cavity's opposite side (see above), gave very high intracavity loss $(\approx 60 \mathrm{~dB})$, i.e. extremely low Q-factor. A cladding mode stripper (CMS) was used to dump the residual pump. Two isolators (ISO1 and ISO2) protected the laser against parasitic reflections from the fiberized optic elements outside the cavity, used for registration laser signal at Outputs 1 and 2.

Laser signals were detected by $5-\mathrm{GHz}$ InGaAs photodetectors (Thorlabs, model DET08CFC), connected to an 8-GHz oscilloscope (LeCroy, model 804HD-MS). CW laser power was always attenuated to $\sim 0.5 \mathrm{~mW}$ to warrant operation of the photodetectors well below saturation. Laser power was measured by a power meter with a thermal head; optical spectra were registered by an optical spectrum analyzer (OSA) (Yokogawa, model AQ6370D).

By $\mathrm{SBS}^{+}$and $\mathrm{SBS}^{-}$in Fig. 1(a) we denote SBS-induced laser waves propagating to the right ("positive") and the left ("negative") directions along the cavity.

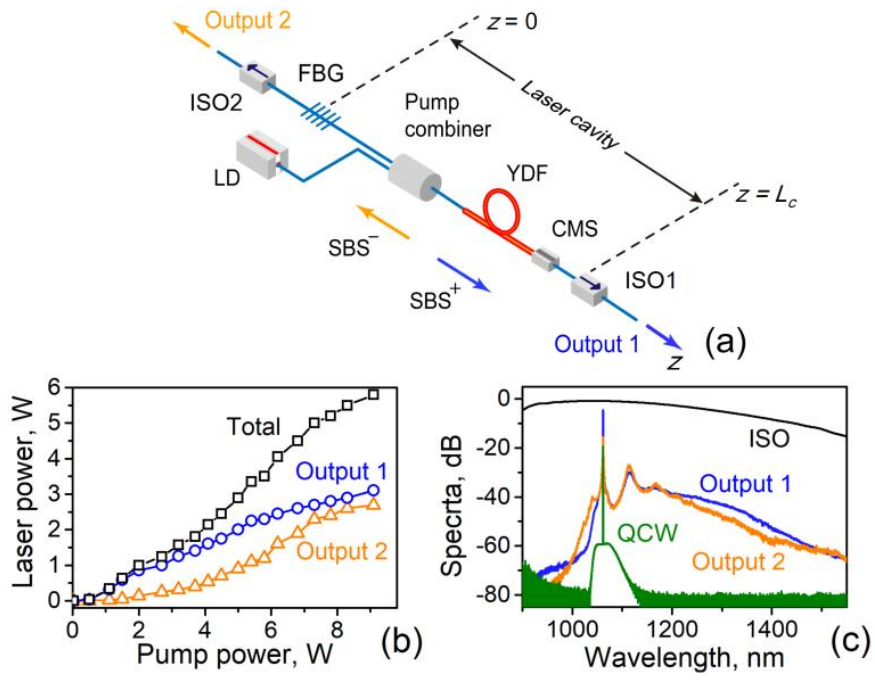

Fig. 1. (a) Experimental setup; $z$ is axis along the laser cavity with zero at FBG position. (b) Laser power vs. pump power: The curves marked as Output 1, Output 2, and Total demonstrate laser power from Output 1, Output 2, and the total power, respectively. (c) Optical spectra. The spectra marked as Output 1 and Output 2 were measured on correspondent laser outputs at $9.1 \mathrm{~W}$ of pump power. The spectrum marked as QCW relates to the laser operation just above the laser threshold (pure QCW) and the one marked as ISO demonstrates the transmission spectrum of the optical isolator.
The laser (QCW) threshold was found to be $\sim 0.7 \mathrm{~W}$ and its overall efficiency, calculated as the ratio of the total power (a sum of powers measured at both laser outputs) to pump power, $P_{P}$, reached $\sim 70 \%$ (see Fig. 1(b)) at pump power of 7 to $8 \mathrm{~W}$. The efficiencies measured separately for Output 1 and Output 2 were by around 2 times lower within this range of pump power. Note that at low pumps $\left(P_{p} \leq 2 \mathrm{~W}\right)$, the efficiency on Output 2 was very low.

The laser spectrum measured just above the threshold demonstrates a narrow peak of QCW lasing (see the curve labeled "QCW" in Fig. 1(c)) above a pedestal of amplified spontaneous emission (ASE). This peak is well fitted with the Gaussian function with FWHM of approximately $40 \mathrm{pm}$ when it is measured by OSA with $32 \mathrm{pm}$ resolution at $1060 \mathrm{~nm}$; after deconvolution, considering that OSA response to a narrow spectrum is Gaussian [15], the spectral width was estimated to be $\sim 24 \mathrm{pm}$. At higher pump powers, the laser spectrum dramatically broadens due to spurious SC, generated in YDF and passive fiber pieces under the action of SBS pulses that get produced in the laser; see the curves labeled "Output 1" and "Output 2" in the figure. Note that these spectra are the result of convolution of the real SC spectra and the transmission ones of the isolators (see the curve labeled "ISO" in Fig. 1(c)); thus, the real SC spectra are even broader.

\section{EXPERIMENTAL RESULTS AND DISCUSSION}

Fig. 2(a) demonstrates an oscilloscope trace of the laser signal collected at $P_{p}=0.73 \mathrm{~W}$ at which the laser operates in QCW mode; the gray curve is the original trace and the blue one is the smoothed signal.

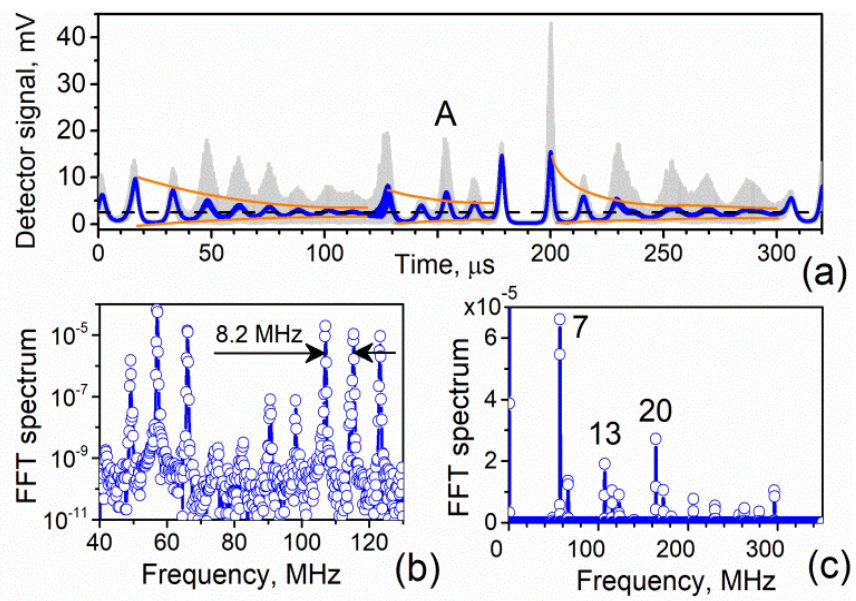

Fig. 2. QCW laser operation. (a) Train of pulses of relaxation oscillations collected at $P_{\text {pump }}=0.73 \mathrm{~W}$. The orange lines demonstrate dumping of relaxation oscillations. The horizontal dash line shows the mean laser power $(300 \mathrm{~mW})$. Pulse A is used for FFT analysis, see plots (b) and (c). (b) A section of FFT spectrum of the pulse A in lin-log scale. The adjacent modebeating peaks are separated by $8.2 \mathrm{MHz}$. (c) FFT spectrum presented in lin-lin scale. The most powerful spectral peaks correspond to beating at laser modes separated by 7, 13, and 20 intermodal intervals.

This laser mode is characterized by pulsing (in fact, by spikes of relaxation oscillations) with pulse mean width at FWHM of $\sim 3 \mu$ s that exceeds by $\sim 25$ times the photon round trip time, $T_{r}(\sim 120 \mathrm{~ns})$; this confirms its operation in QCW. 
Note that some QCW spikes are by a few orders higher than the mean $\mathrm{CW}$ power: compare the instant pulse arisen at 200 $\mu$ s and the mean laser signal, shown in the figure by the dashed black line.

From the FFT spectrum taken for spike A (see Figs. 2(b) and (c)), it is seen that mode-beating frequency is $\sim 8.2 \mathrm{MHz}$ and that laser signal is composed of several longitudinal modes with the most powerful spectral components displaced by 7, 13, and 20 intermodal intervals (certainly, each QCW spike is represented by a unique set of such components).

As mentioned above, the two operation sub-regimes, QCW and SBS, coexist at pump powers above the threshold. This feature was confirmed by a study proceeded over a set of long (10-ms) traces of laser signal, collected for the range of pump powers varied from the threshold (when QCW sub-regime prevails) up to $9.1 \mathrm{~W}$ (when the laser oscillates in the subregime of very stable SBS pulses, almost free from any insertion of QCW sub-regime windows). Three examples of such traces are shown in Fig. 3 (for simplifying visualization of pulsing structure, we provide here their 1-ms fragments).

From Fig. 3, it is seen that SBS pulses are much greater in magnitude than QCW spikes and that trains of SBS pulses are alternated randomly by windows of QCW sub-regime. Such windows (i.e. intervals between the last in train SBS pulse and the first QCW pulse) have frames approximately equal to the mean interval between SBS pulses. Upon pump level, the duration of QCW windows varies in the range from a fraction to a large number of the mean interval between SBS pulses.

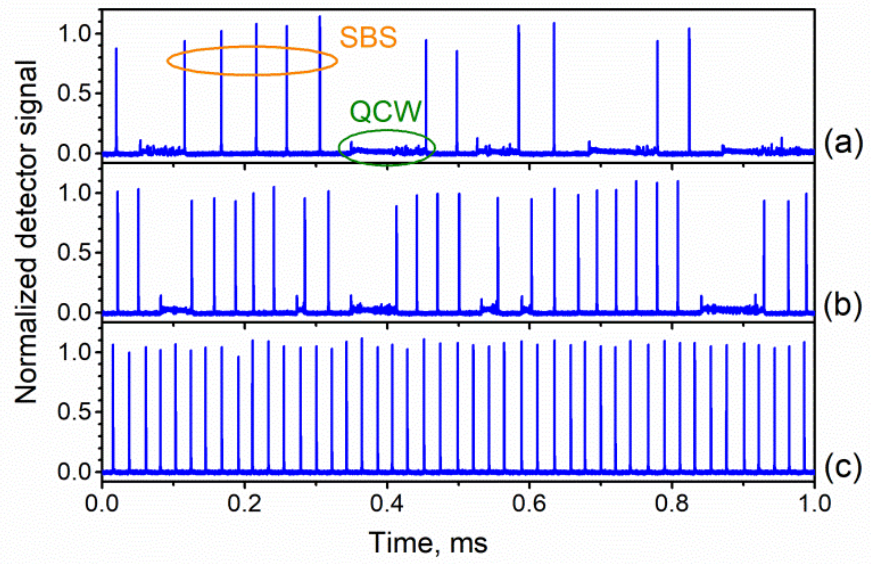

Fig. 3. Traces of the laser signal collected from Output 1 for pump powers (a) $3.8 \mathrm{~W}$, (b) $5.0 \mathrm{~W}$, and (c) $6.2 \mathrm{~W}$. Olive and orange ellipses mark one of QCW intervals and one of the trains of pure SBS pulses, respectively.

An example of QCW sub-regime intermitting trains of regular SBS pulses (I, II, III etc.) is shown in lin-log scale in Fig. 4(a) after smoothing. It is seen that pulse A next to SBS pulse II is much smaller in magnitude than SBS pulses; see also Fig. 4(b) where pulse A is plotted in zoomed lin-lin scale. The pulse comprises a spike of relaxation oscillations (its Gaussian fit is denoted as "1") and a small SBS-like spike (denoted as "2") that is incapable to develop in a regular SBS pulse; FWHMs of the spikes differ by 15 times $(\sim 300 \mathrm{~ns}$ and $\sim 20 \mathrm{~ns}$, respectively).
Note that, at the relevant to Fig. 4 high pump power $\left(P_{p}=\right.$ $7.9 \mathrm{~W}$ ), width of QCW pulses is lesser by one order as compared the case of low pump powers (refer to Fig. 2) but considerably larger (by $\sim 2.5$ times) than $T_{r}$. In contrast, regular SBS pulses (I, II, III etc.) are by $\sim 2$ orders of magnitude larger than $\mathrm{QCW}$ pulses.

Furthermore, Fig. 4(c) shows a SBS pulse (denoted as II) arisen just after terminating the QCW window (lasting by $\Delta t_{Q C W} \sim 5 \mu \mathrm{s}$ : refer to Fig. 4(a)). Its shape (as those of other SBS pulses) is irregular as such pulses are in fact produced by SBS processes of multiple orders and of random polarizations. Note that such a "delayed" SBS pulse develops instead of a regular SBS one given that the seed for the latter (refer to spike "2" in Fig. 4(b)) has insufficient spectral density (due to a large number of longitudinal modes of which it consists) and, probably, because of partly faded inversion at enrolling and fluctuations of polarization state of the laser wave, influencing the SBS pulses threshold [28].
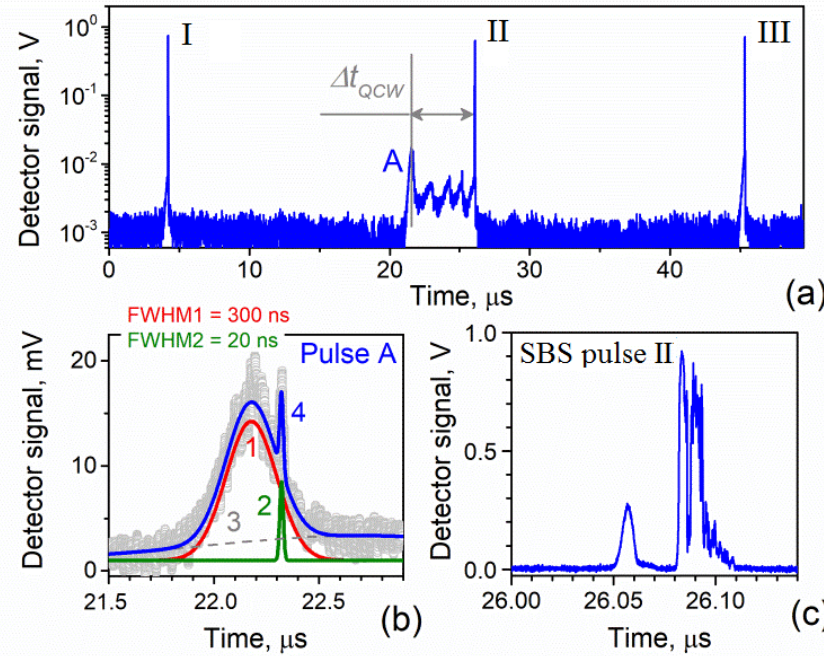

Fig. 4. (a) Section of the train of the smoothed SBS pulses that contains a short interval of QCW lasing in lin-log scales. Pump power is 7.9 W. Pulses I, II, and III are regular SBS pulses, pulse A is QCW pulse. (b) Pulse A shown in the zoomed lin-lin scales. Circles are experimental points, lines 1 (red) and 2 (olive) are the Gaussian fits of relaxation oscillation' spike and of small SBS pulse, respectively, dash line 3 fits change in the bias level, and line 4 (blue) is the best cumulative fit. (c) SBS pulse II.

The ratio of the sum of all QCW windows encountered during a long oscilloscope trace, $t_{Q C W}=\Sigma\left(\Delta t_{Q C W}\right)$, to the length of the trace $(T=10 \mathrm{~ms})$ was used further to calculate the probability of QCW sub-regime as $t_{Q C W} / T$; accordingly, the probability of SBS sub-regime was found as $1-\left(t_{Q C W} / T\right)$. These probabilities are plotted in Fig. 5(a). 

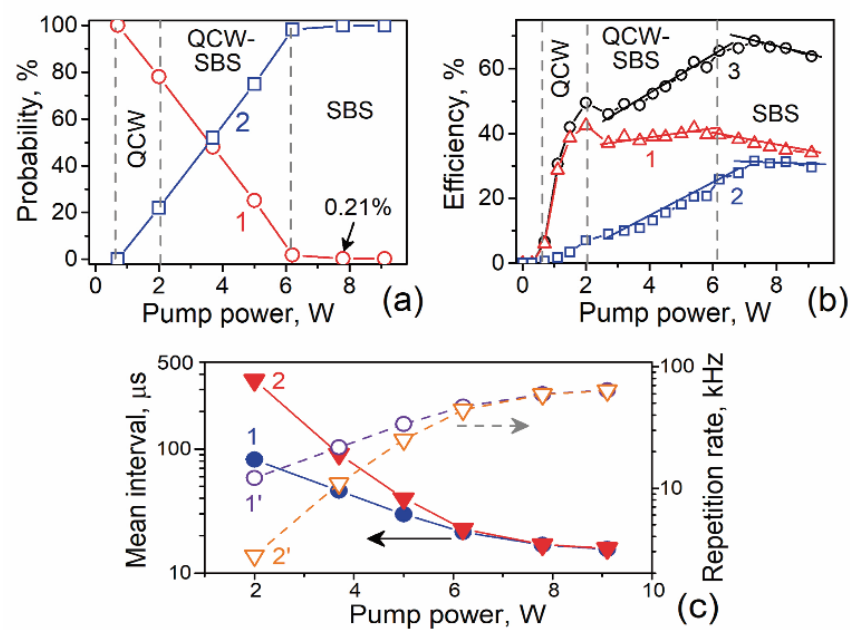

Fig. 5. (a) Probability of QCW (curve 1) and SBS (curve 2) pulsing. (b) YDFL efficiency $v s$. pump power: curves 1 and 2 correspond to laser outputs 1 and 2, respectively; curve 3 demonstrates the total laser efficiency. The vertical dash lines separate areas of mostly QCW, mixture of QCW and SBS, and mostly SBS lasing. (c) Mean interval between SBS pulses (the left scale) and repetition rate of the pulses (the right scale) $v s$. pump power. In all plots symbols are experimental points.

As seen, the probability of QCW sub-regime decreases from $100 \%$ close to laser threshold $\left(P_{p} \approx 0.7 \mathrm{~W}\right)$ to fractions of percent at high pump powers $\left(P_{p}>6 \mathrm{~W}\right)$; on the contrary, the probability of SBS sub-regime increases from 0 up to nearly $100 \%$ with increasing pump power but never reaches $100 \%$ (at least in the range of pump powers accessible using our setup).

The partial laser efficiencies, measured as the ratios of laser powers at Outputs 1 and 2 to pump power, are presented in Fig. 5(b) by curves 1 and 2. In turn, the overall laser efficiency (the sum of the partial two) is shown in the figure by curve 3 . As seen, three basins exist in this plot, differing by the slope efficiency (see also Fig. 1(b)), which can be characterized as follows:

(i) At low pump powers $\left(P_{p}<2 \mathrm{~W}\right.$, QCW basin, where QCW sub-regime dominates), the efficiency at Output 1 strongly increases with increasing pump power; in turn, the efficiency at Output 2 is approximately an order lower. This occurs because of strong asymmetry in the laser cavity, given that reflection from isolator 1 is by $40 \mathrm{~dB}$ less than that from FBG. Note that this kind of behavior is explained in terms of two contra-propagating waves' laser model [29], applicable to QCW sub-regime whose contribution at Output 2 is by two orders less than at Output 1. That is, growth of laser power (and efficiency) at Output 2 is mostly due to increasing probability of SBS sub-regime.

(ii) At medium pump powers ( $2 \mathrm{~W}$ to $6 \mathrm{~W}$, QCW-SBS basin, where contributions of QSW and SBS sub-regimes are comparable), the efficiency at Output 1 is virtually constant, whereas that at output 2 rises with almost unchanged slope. This occurs due to further decreasing probability of QCW subregime to intermit SBS pulsing (see Fig. 5(a)) and linear growth of probability of SBS sub-regime.

(iii) At the highest pump powers (> 6W, SBS basin, where SBS sub-regime dominates), the overall YDFL efficiency reaches maximum $(\sim 70 \%)$ at $P_{p} \sim 7$ to $8 \mathrm{~W}$; however, beyond this value the slopes of all the efficiencies become negative.
The latter happens due to prevailing in the laser output of SBS pulses that effectively generate broadband SC (refer to Fig. $1(\mathrm{c}))$, the most of spectral components of which are suppressed by band-pass optical filtering inside the isolators (see Fig. 1(c)) and hence get less detectable.

An interesting observation concerning the competition of QCW and SBS pulsing (Fig. 5(a)) relates to the influence of pump depletion to the probability of each of these two regimes. Considering the known parameters of YDF (absorption cross-section of $\mathrm{Yb}^{3+}$ ion at the pump wavelength $\left(\sim 2.5 \times 10^{-24} \mathrm{~m}^{2}\right)$, the relaxation time of $\mathrm{Yb}^{3+}(\sim 1 \mathrm{~ms})$, MFD $(6.5 \mu \mathrm{m})$, and also the pump to fiber core overlap factor $(\sim 0.0026)$ estimated by us), the saturation power $\left(P_{\text {sat }}\right)$ is found to be $\sim 400 \mathrm{~mW}$. Just above the laser threshold, the residual pump $P_{\text {res }} \approx 55 \mathrm{~mW}$ is much less than $P_{\text {sat }}$, which result in the laser operation in QCW regime. At pump power above $6 \mathrm{~W}$ no pump depletion is observed since $P_{\text {res }}>P_{\text {sat }}$; in such circumstances practically pure SBS pulsing occurs. With increasing pump from laser threshold to $6 \mathrm{~W}$, pump depletion monotonously decreases, leads to increasing probability of SBS pulsing and decreasing probability of QCW regime.

Another important detail relates to periodicity of SBS pulses. In Fig. 5(c), are shown two sets of dependences allowing insight to how the mean intervals between adjacent SBS pulses and their repetition rate behave at varying pump power.

The first set of dependences (curves 1 and 2: see filled symbols connected by solid lines) reveal decreasing of the mean interval, $\Delta T_{\text {mean }}$, between SBS pulses with increasing pump power. Note that, whereas curve 1 was obtained as the mean value of the intervals between SBS pulses when no CQS windows arisen (and hence these windows were simply not accounted for), curve 2 was obtained as formal ratio of the length of a long trace of laser signal and the number of SBS pulses within it minus one. As seen, these two dependencies, measured applying different methods, differ strongly at low pump powers but almost merge at high pump powers. Accordingly, the second set of dependences (curves 1' and 2') stand for repetition rates of SBS pulses; these dependences were simply calculated as the reciprocals for the correspondent mean intervals and thus demonstrate the opposite trends. The shortening of the mean interval between SBS pulses (the enlarging of the repetition rate) with increasing pump seems to follow an increase of the rate of charging YDF to some value of population inversion in the system of $\mathrm{Yb}^{3+}$ ions, permitting enough gain for generating succeeding SBS pulses.

Photon statistics measured for both pure QCW and stable SBS pulsing demonstrate quite different behavior, see Fig. 6. In each plot of this figure both axes are normalized to the maximum of photon count (the vertical axis) or to the mean laser power $\left(P_{\text {mean }}\right)$ (the horizontal axis). 

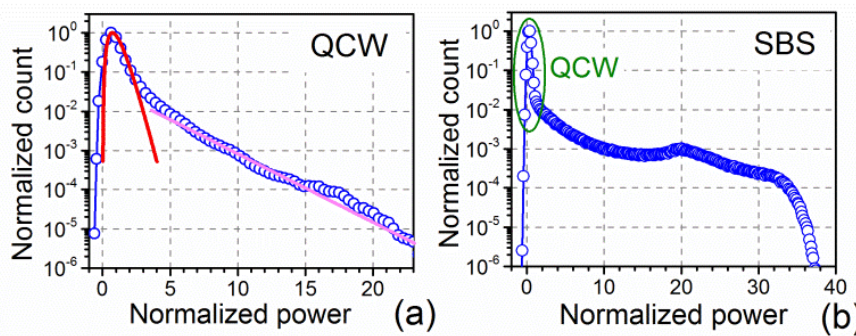

Fig. 6. (a) Normalized photon count of (a) QCW and (b) SBS pulsing obtained from the original trains of laser signals at pump power of $0.73 \mathrm{~W}$ and $7.2 \mathrm{~W}$, respectively. In both cases the laser signals at the detector input were polarized.

From Fig. 6(a) one can see that the histogram of photon counts of pure QCW laser signal consist of two parts. One of them (the upper part) corresponds to the level to which relaxation oscillations damps out into $\mathrm{CW}$ oscillations; it is centered approximately at a half of the mean laser power. This part may be approximated by Bose-Einstein distribution (red line) with a number of independent states of amplified spontaneous emission $M=3.7$ [15], which is determined by $\mathrm{CW}$ optical spectrum width (24 pm, see above) and RF band of registration devices used ( $2.5 \mathrm{GHz}$ in this case). The tail of the histogram above $4 P_{\text {mean }}$ can be fit with exponential decay (linear in lin-log scale, magenta line) with absolute slope lower than the slope that can be attained using Bose-Einstein distribution.

Fig. 6(b) shows histogram of photon count of pure SBS pulsing. It also consists of two parts: the upper narrow part at laser power $\leq P_{\text {mean }}$ corresponds to $\mathrm{CW}$ operation observed between SBS pulses and another one, above $P_{\text {mean }}$, is characterize by very low slope and describes statistics of SBS pulses. It is seen that the histogram of SBS pulses dramatically drops down at laser power above $\sim 32 P_{\text {mean }}$ (cut-off power) due to nonlinear effects resulting in strong broad-band SC generation (see Fig. 1(c)).

The detailed statistical properties of SBS pulses are illustrated in Fig. 7, where the main plots in panels (a) and (b) stand for the distributions of the experimental events presented in the space $(\delta T, \delta A)$, where $\delta T=\Delta T / \Delta T_{\text {mean }}$ and $\delta A=A / A_{\text {mean }}$ are the normalized deviations of the intervals between SBS pulses and their magnitudes, respectively $\left(A_{\text {mean }}\right.$ is the mean magnitude of SBS pulses).
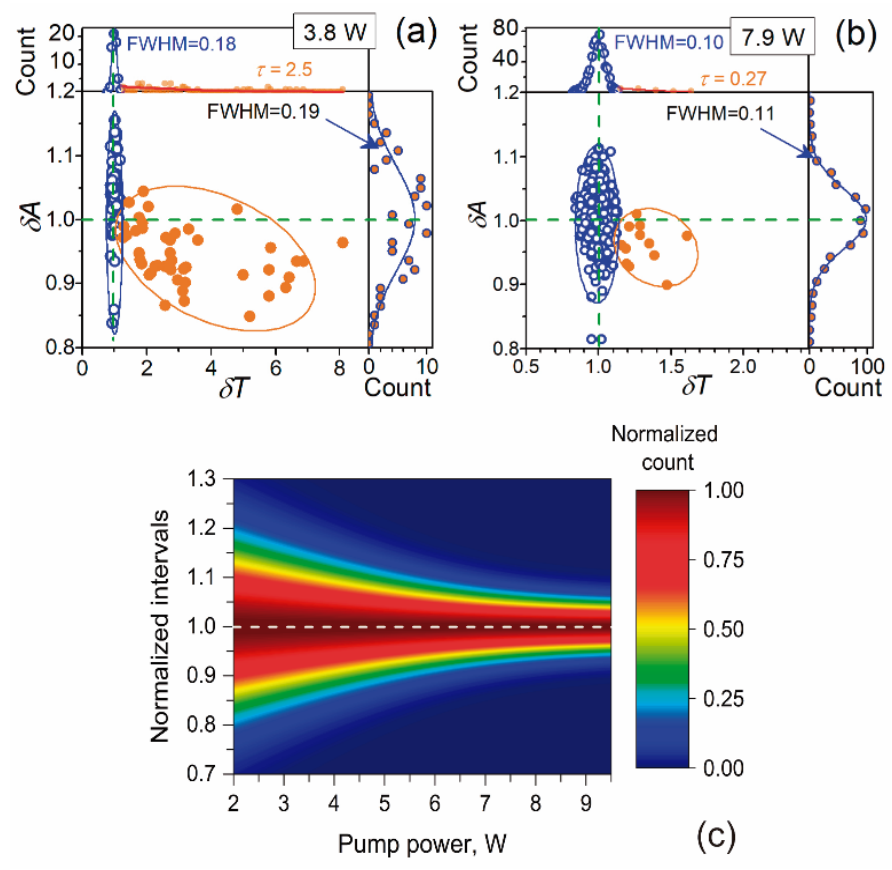

Fig. 7. (a) and (b): The main plots: experimental points shown in the space $(\delta T, \delta A)$. The right insets are histograms of $\delta A$ (made for data shown by the open and filled symbols counted together); the upper insets are histograms of $\delta T$ (made separately for the open and filled symbols). Pump power is marked on top of the graphs. (c) Color map of Gaussian distribution of the normalized intervals between SBS pulses as a function of pump power.

Naturally, these two variables $(\delta T, \delta A)$ can serve the measures for timing and amplitude jitters in the system. Note that pump powers at which the data were collected are indicated in the right upper corners of both panels. The empty and filled symbols stand, correspondingly, for the cases of absence and presence of QCW sub-regime. On the right sides of the main plots are shown the histograms of $\delta A$ fitted by the Gaussian distributions and on their tops - the histograms of $\delta T$ fitted by the Gaussian function (only empty symbols) and by the exponential decay function (only filled symbols). Note that FWHMs of the Gaussian fits for $\delta A$ and $\delta T$ distributions are virtually equal each to other at the same pump level.

It is seen from Fig. 7 that the empty blue symbols (standing for the case of QCW sub-regime absence) are grouping within concise circles (seen as ellipses due to different scale factors of the axes) with diameters dependent on pump power (the higher pump level, the smaller the diameter). For instance, FWHMs of the Gaussian fits of the relevant histograms drop down by approximately two times with increasing pump power from $3.8 \mathrm{~W}$ to $7.9 \mathrm{~W}$. The main part of the orange filled symbols (standing for the case of QCW sub-regime presence) are grouping within the concise ellipses located in the lower right sectors of the panels; thus, magnitudes of the majority of the SBS pulses that arise just after closing the windows of QCW sub-regime are less than the mean values.

Note that the right tails of $\delta T$-histograms, inherent to the intervals containing QCW-windows, are characterized by the decay constant, $\tau$, as a function of pump power: $\tau$ decreases by one order of magnitude, from 2.5 to 0.27 , when pump power is increased from $3.8 \mathrm{~W}$ to $7.9 \mathrm{~W}$. Considering $T_{\text {mean }}$ for these 
two pump powers from line 1 in Fig. 5(c) $(46 \mu$ s and $17 \mu \mathrm{s}$ respectively), one can obtain that the major part of the intervals is less than $115 \mu \mathrm{s}$ in the first case and $4.5 \mu \mathrm{s}$ in the second one. In other words, the upper limit of the intervals with QCW decreases by 25 times with increasing pump power from $3.8 \mathrm{~W}$ to $7.9 \mathrm{~W}$. This observation is consistent with the global tendency of lowering the probability of QCW subregime with pump power growth (refer to Fig. 5(a)).

The color diagram in Fig. 7(c) serves to characterize a decrease of timing jitter of SBS pulses with increasing pump power in the broad range of the latter. This diagram was obtained after fitting all histograms of $\delta T$-distributions by the Gaussian functions with posterior normalizing by maxima of these functions. One sees from this diagram that width of the distributions decreases by $\sim 3$ times at varying pump power from $2 \mathrm{~W}$ to $9.5 \mathrm{~W}$. At the maximal pump power, FWHM of the histogram is close to 0.1 ; thus, in this case the intervals between SBS pulses fluctuate within the range $\pm 5 \%$ of their mean value $\left(\Delta T_{\text {mean }}\right)$.

One of the most important features of SBS lasing relates to contribution of multi-order Stokes components in SBS pulse evolution, the effect recently theoretically studied in ref. [30],[31] and experimentally observed in a multi-pass YDF based amplifier, seeded by a DFB laser; see ref. [27].

To address the issue, consider distribution of $\mathrm{CW}$ laser power along the cavity (see Fig. 8(a)), found in the frames of the contra-propagating waves model at fixed gain in an YDF [29]. Note that since the pulses in QCW sub-regime are long enough, the steady-state $(\mathrm{CW})$ approximation is acceptable.

At QCW, YDF round-trip gain equals to the cavity roundtrip loss (60 dB in our case); accordingly, one-pass gain is 30 $\mathrm{dB}$. Thus, the powers that bear the two contra-propagating waves 1 and 2 (propagating in opposite directions: refer to Fig. 1(a)) get gain of $30 \mathrm{~dB}$ each during a single pass through the 8-m YDF. According to the boundary conditions, intracavity power drops by $10 \mathrm{~dB}$ after reflection by $\mathrm{FBG}(z=0)$ and by $50 \mathrm{~dB}$ after reflection from the isolator $\left(z=L_{c}\right)$; see layout of the process in Fig. 8(a).

As seen from Fig. 8(a), the laser reaches the highest power in the fiber section located close to isolator ISO1 when travelling in the "positive" direction (wave 1); this section is highlighted by the green ellipse in the figure. When this wave reaches the intensity equal to SBS threshold [32], it starts to produce a reflected SBS-wave in the opposite ("negative") direction at the wavelength shifted by $\sim 60 \mathrm{pm}$ (the $1^{\text {st }}$ Stokes component), which, in its turn, produces another SBS-wave at the wavelength shifted by another $60 \mathrm{pm}$ (the $2^{\text {nd }}$ Stokes component), and so on. Since YDF is charged to some, relatively high, inversion level, permitting a high enough gain to ignite and boost SBS generation, each SBS wave occurs in the form of a short (nanosecond) pulse. Certainly, such SBS pulses arisen as the odd Stokes waves propagate in the negative $z$-direction (to Output 2) and those arisen as the even Stokes waves travel in the positive $z$-direction (to Output 1). This scenario is confirmed by the optical spectra collected at the opposite laser outputs, as seen from Fig. 8(b).
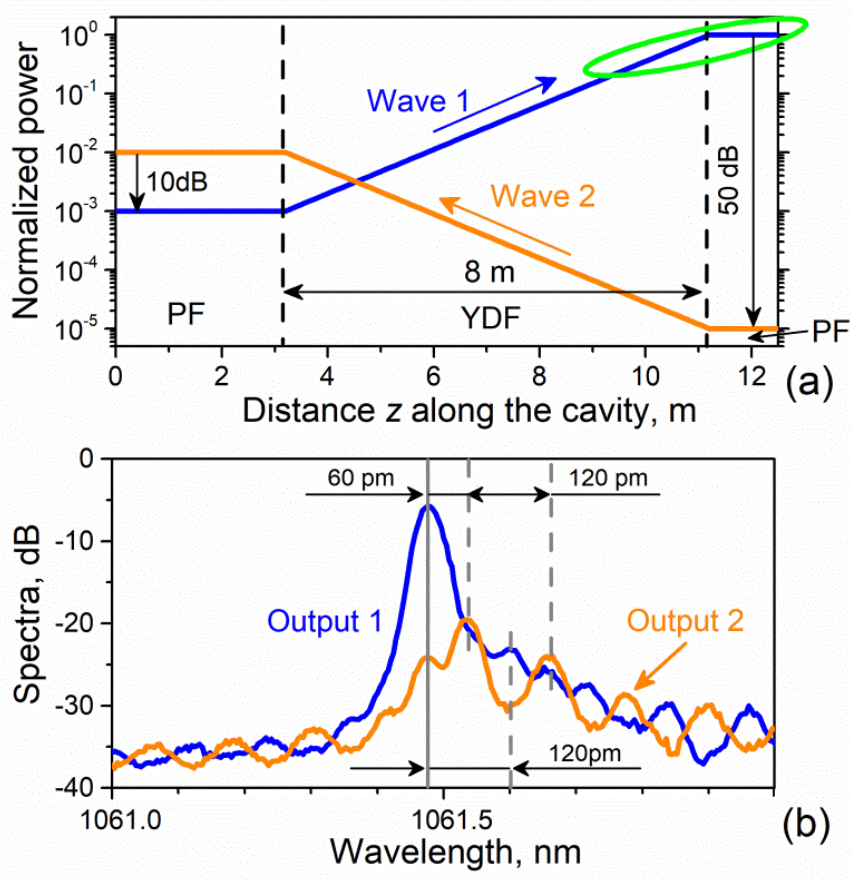

(b)

Fig. 7. (a) Sketch of distribution of the laser wave intensity along the laser cavity. Zero-distance corresponds to FBG position. Areas indicated as PF correspond to sections of passive fiber. (b) Optical spectra of the laser signal measured on both laser outputs. Vertical gray solid line indicates the laser wavelength. OSA resolution is $32 \mathrm{pm}$.

From this figure, it is seen that both spectra demonstrate peaks at the laser wavelength $(1061.4 \mathrm{~nm})$ and, also, a cascade of peaks separated from the laser wavelength by the integer number of Stokes shifts. The peaks at the laser wavelength related to narrowband QCW operation differ in magnitude by approximately $20 \mathrm{~dB}$ that coincides with the difference in power of the two laser waves shown in Fig. 8(a): power of wave 1 at $z=L_{c}$ is by two orders higher than that of wave 2 at $z=0$. It is worth to mention that the most of laser power at the laser wavelength is contained in the windows of QCW subregime, sporadically arisen between the trains of SBS pulses (refer to Fig. 3); it is the reason of why power at this wavelength is a small part of the laser signal at Output 2. Furthermore, the spectrum measured at Output 2 (orange curve) comprises a set of peaks of the odd Stokes orders $\left(1^{\text {st }}\right.$, $3^{\text {rd }}, 5^{\text {th }}$ etc.) whereas that measured at Output 1 (blue curve) comprises mostly the peaks of the even Stokes orders $\left(2^{\text {nd }}, 4^{\text {th }}\right.$, $6^{\text {th }}$ etc.). Note that the peaks of the $3^{\text {rd }}$ and $5^{\text {th }}$ orders arise due to small reflections of the correspondent components of wave 2 by the FBG's lateral spectral peaks. It is interesting that the spectra of laser signals measured at Outputs 1 and 2 reveal the generation of SBS pulses at the anti-Stokes wavelengths, too: the even and the odd ones at Output 1 and Output 2, respectively. However, their magnitudes are by an order less than those at the Stokes components. Note that a similar pattern of spectral response due to SBS process was recently reported in Ref. [27] for a multi-pass fiber amplifier seeded by a single-frequency laser. We also should note that using a broadband (chirped) FBG as a selective reflector of the laser cavity will result in broadening QCW laser spectrum so that some important spectral details as SBS orders will be 
indistinguishable. Moreover, since YDFL with spectrally broad cavity reflectors suffers from the spectral modes' selfsweeping [33], the effect of SBS pulses' jitter may be enhanced.

\section{CONCLUSION}

In this paper, we discussed the featuring sides of operation of a double-clad YDFL with very high intra-cavity loss $(\sim 60$ $\mathrm{dB})$. We demonstrated that in such type of laser two pulsed sub-regimes, QCW and SBS-ignited SQS, coexist at any pump power. With increasing the pump power, the probability of QCW sub-regime fades from 100\% (beyond the threshold, $\sim 0.7 \mathrm{~W}$ ) to $\sim 0.2 \%$ (at maximum pump power, $9.1 \mathrm{~W}$ ) whereas the probability of the SBS-SQS sub-regime grows from $\sim 0 \%$ to $\sim 99.8 \%$. Furthermore, apart characterization of the QCW and SBS-SQS sub-regimes, we demonstrated strong broadband supercontinuum (SC) generation from the same laser, mostly enrolling beyond the laser wavelength, resulted from the nonlinear effects in YDF and passive fiber (both with normal dispersion).

We also discussed the results of a statistical analysis of timing and amplitude jitters of SBS pulses. We showed that the experimental points representing the intervals between SBS pulses and pulse magnitudes (these two variables are the measures of the timing and amplitude jitters) are grouping in the space within two areas, inherent to strongly different distributions. Specifically, the points relating to the intervals of "pure" SBS pulsing are described by the Gaussian distribution whereas the points corresponding to stochastically intermitting windows of QCW sub-regime obey the exponential law of distribution. It is important that the width of the distribution of the intervals with windows of QCW subregime is much broader (by an order of magnitude) than that of the intervals inherent to pure SBS pulsing. With increasing pump power, SBS pulses become more stable, with the relevant distribution of FWHM widths being as small as $10 \%$.

We demonstrated then that the YDFL generates two series of SBS pulses that selectively propagate in two different directions: the ones born by SBS at the even Stokes orders travel in the cavity in one side while the ones born by SBS at the odd Stokes orders in the opposite side. The propagation direction obeyed by the different Stokes components is eventually defined by the boundary conditions of the cavity: the odd Stokes waves select the direction in which the cavity coupler "works worse" (it is the reflection from inside the isolator, of $\sim-50 \mathrm{~dB}$ ) whereas the even Stokes waves choose the direction of better reflection (from the FBG-coupler, of $-10 \mathrm{~dB})$.

The reported results are useful for in-depth understanding of the physics behind SBS-SQS pulsing in YDFLs with low Qfactor cavities, which, in turns, may be useful for a line of applications, including development of simple and cheap sources of nanosecond pulses and their use as pump for broadband supercontinuum generation and rogue waves.

\section{REFERENCES}

[1] D. J. Richardson, J. Nilsson, and W. A. Clarkson, "High power fiber lasers: Current status and future perspectives [Invited]," J. Opt. Soc. Amer. B, vol. 27, no. 11, pp. B63-B92, Nov. 2010.

[2] Y. Jeong, J. K. Sahu, D. N. Payne, and J. Nilsson, "Ytterbium-doped large-core fiber laser with $1.36 \mathrm{~kW}$ continuous-wave output power," Opt. Express, vol. 12, no. 25, pp. 6088-6092, Nov. 2004.

[3] J. Zheng, W. Zhao, B. Zhao, C. Hou, Z. Li, G. Li, Q. Gao, P. Ju, W. Gao, S. She, P. Wu, and W. Li, " $4.62 \mathrm{~kW}$ excellent beam quality laser output with a low-loss $\mathrm{Yb} / \mathrm{Ce}$ co-doped fiber fabricated by chelate gas phase deposition technique," Opt. Mater. Express, vol. 7, no. 4, pp. 1259-1266, Apr. 2017.

[4] A. V. Kir'yanov, M. Paul, Y. O. Barmenkov, S. Das, M. Pal, and L. Escalante-Zarate, "Yb ${ }^{3+}$ concentration effects in novel $\mathrm{Yb}$ doped lanthano-alumino-silicate fibers: experimental study," IEEE Quant. Electron., vol. 49, pp. 528-544, 2013.

[5] H. Gebavi, S. Taccheo, D. Milanese, A. Monteville, O. Le Goffic, D. Landais, D. Mechin, D. Tregoat, B. Cadier, and T. Robin, "Temporal evolution and correlation between cooperative luminescence and photodarkening in ytterbium doped silica fibers," Opt. Express, vol. 19, pp. 25077-25083, 2011.

[6] A. D. Guzman Chavez, A. V. Kir'yanov, Y. O. Barmenkov, and N. N. Il'ichev, "Reversible photo-darkening and resonant photobleaching of Ytterbium-doped silica fiber at in-core 977-nm and 543-nm irradiation," Las. Phys. Lett., vol. 4, pp. 734-739, 2007.

[7] S. Jetschke, A. Schwuchow, S. Unger, M. Leich, M. Jäger, and J. Kirchhof, "Deactivation of $\mathrm{Yb}^{3+}$ ions due to photodarkening," Opt. Mater. Express, vol. 3, pp. 452-458, 2013.

[8] Y. Feng, B. M. Zhang, J. Nilsson, "Photodarkening-Induced Phase Distortions and Their Effects in Single-Channel and Coherently Combined Yb-Doped Fiber Chirped Pulse Amplification Systems," J. Lightw. Technol., vol. 36, no. 23, pp. 5521-5527, Dec. 2018.

[9] H. Li, L. Zhang, R. Sidharthan, D. Ho, X. Wu, N. Venkatram, H. Sun, T. Huang, and S. Yoo, "Pump Wavelength Dependence of Photodarkening in Yb-Doped Fibers", J. Lightw. Technol., vol. 35, no. 13, pp. 25352540, Jul. 2017.

[10] N. Zhao, W. Li, J. Li, G. Zhou, J. Li, "Elimination of the Photodarkening Effect in an Yb-Doped Fiber Laser with Deuterium," $J$. Lightw. Technol., vol. 37, no. 13, pp. 3021-3026, Jul. 2019.

[11] Y. Wang, C. Gao, X. Tang, H. Zhan, K. Peng, L. Ni, S. Liu, Y. Li, C. Guo, X. Wang, L. Zhang, J. Yu, L. Jiang, H. Lin, J. Wang, F. Jing, and A. Lin, "30/900 Yb-doped aluminophosphosilicate fiber presenting 6.85$\mathrm{kW}$ laser output pumped with commercial 976-nm laser diodes," $J$. Lightw. Technol., vol. 36, no. 16, pp. 3396-3402, Aug. 2018.

[12] M. N. Zervas and C. A. Codemard, "High Power Fiber Lasers: A Review," IEEE J. Sel. Topics Quantum Electron., vol. 20, no. 5, art. no. 0904123, Sept./Oct. 2014.

[13] A. Ferin, V. Gapontsev, V. Fomin, A. Abramov, M. Abramov, and D. Mochalov, "31.5 kW CW fiber laser with $100 \mu \mathrm{m}$ delivery," presented in 15th International Conference "Laser Optics 2012", St. Petersburg, Russia, 26-28 June 2012, paper TuSY1-1.4.

[14] E. A. Shcherbakov, V. V. Fomin, A. A. Abramov, A. A. Ferin, D. V. Mochalov, and V. P. Gapontsev, "Industrial grade $100 \mathrm{~kW}$ power CW fiber laser," in Advanced Solid-State Lasers Congress Technical Digest, San Diego, California, USA, 29 Jan. - 1 Feb. 2013, paper ATh4A.2.

[15] P. Muniz-Cánovas, Y. O. Barmenkov, A. V. Kir'yanov, J. L. Cruz, and M. V. Andrés, "Ytterbium-doped fiber laser as pulsed source of narrowband amplified spontaneous emission," Sci. Rep., accepted for publication.

[16] A. E. Bednyakova, O. A. Gorbunov, M. O. Politko, S. I. Kablukov, S. V. Smirnov, D. V. Churkin, M. P. Fedoruk, and S. A. Babin, "Generation dynamics of the narrowband Yb-doped fiber laser," Opt. Express, vol. 21, no. 7, pp. 8177-8182, Mar. 2013.

[17] J. W. Goodman, Statistical Optics. New York: Wiley, 2000, ch. 6.

[18] S. V. Chernikov, Y. Zhu, J. R. Taylor, and V. P. Gapontsev, "Supercontinuum self-Q-switched ytterbium fiber laser," Opt. Lett., vol. 22, no. 5, pp. 298-300, May 1997.

[19] L. Pan, I. Utkin, R. Lan, Y. Godwal, and R. Fedosejevs, "High-peakpower subnanosecond passively Q-switched ytterbium-doped fiber laser," Opt. Lett., vol. 35, no. 7, pp. 895-897, Jul. 2010.

[20] A. V. Kir'yanov, Y. O. Barmenkov, and M. V. Andres, "An experimental analysis of self-Q-switching via stimulated Brillouin scattering in an ytterbium doped fiber laser," Las. Phys. Lett., vol. 10, no. 5, art. no. 055112, May 2013. 
[21] X. Luo, Z. Xu, J. Peng, L. Yang, N. Dai, H. Li, and J. Li, "Simple opencavity pulsed Brillouin fiber laser with broadband supercontinuum generation," Appl. Phys. B, vol. 123, no. 10, art. no. 259, Oct. 2017.

[22] P.-H. Hanzard, M. Talbi, D. Mallek, A. Kellou, H. Leblond, F. Sanchez, T. Godin, and A. Hideur, "Brillouin scattering-induced rogue waves in self-pulsing fiber lasers," Sci. Rep., vol. 7, art. no. 45868, Apr. 2017.

[23] D. Hua, J. Su, W. Cui, Y. Yan, and P. Jiang, "All-fiberized SBS-based high repetition rate sub-nanosecond $\mathrm{Yb}$ fiber laser for supercontinuum generation," Las. Phys. Lett., vol. 11, no. 12, art. no. 125103, Dec. 2014.

[24] R. Ma, Y. J. Rao, W. L. Zhang, X. Zeng, X. Dong, H. Wu, and Z. N. Wang, "Backward supercontinuum generation excited by random lasing," IEEE J. Sel. Topics Quantum Electron., vol. 24, no. 3, art. no. 0901105, May/Jun. 2014.

[25] J. Gao, T. Ge, W. Li, H. Kuang, and Z. Wang, "GHz high power Ybdoped picosecond fiber laser and supercontinuum generation," Appl. Opt., vol. 53, no. 36, pp. 8544-8548, Dec. 2014.

[26] N. Wang, J.-H. Cai, X. Qi, S.-P. Chen, L.-J. Yang, and J. Hou, "Ultraviolet-enhanced supercontinuum generation with a mode-locked Yb-doped fiber laser operating in dissipative-solitonresonance region," Opt. Express, vol. 26, no. 2, pp. 1689-1696, Jan. 2018.

[27] Z. Pan, L. Meng, Q. Ye, H. Cai, Z. Fang, and R. Qu, "Repetition rate stabilization of the SBS Q-switched fiber laser by external injection," Opt. Express, vol. 17, no. 5, pp. 3124-3129, Feb. 2009.

[28] R. H. Stolen, "Polarization Effects in Fiber Raman and Brillouin Lasers," IEEE Quant. Electron., vol. 15, no. 10, pp. 1157-1160, Oct. 1979.

[29] I. Kelson and A. Hardy, "Optimization of strongly pumped fiber lasers," J. Lightw. Technol., vol. 17, no. 5, pp. 891-897, May 1999.

[30] S. Hou, Y. Lou, N. Zhao, P. Chen, F. Zhang, Y. Chen, F. Lin, J. Li, L. Yang, J. Peng, H. Li, and N. Dai, "Robust Q-switching based on stimulated Brillouin scattering assisted by Fabry-Perot interference," Opt. Express, vol. 27, no. 4, pp. 5745-5754, Feb. 2019.

[31] D. Boukhaoui, D. Mallek, A. Kellou, H. Leblond, F. Sanchez, T. Godin, and A. Hideur, "Influence of higher-order stimulated Brillouin scattering on the occurrence of extreme events in self-pulsing fiber lasers," Phys. Rev. A, vol. 100, no. 1, art. no. 013809, Jul. 2019.

[32] G. P. Agrawal, Nonlinear Fiber Optics. 3rd ed., San Diego: Academic Press, 2001.

[33] A.V. Kir'yanov and N.N. Il'ichev, "Self-induced laser line sweeping in an ytterbium fiber laser with non-resonant Fabry-Perot cavity," Las. Phys. Lett., Lett. Vol. 8, no. 4, 305-312, Apr. 2011.

Pablo Muniz-Cánovas received his B.S. degree in Electronics and Communications Engineering from the University of Guanajuato, Guanajuato, Mexico, in 1998, and M.D. in Information Technology from Autonomous Institute of Technology, Mexico City, Mexico, in 2005. He worked for 17 years in private sector in TICs companies such as Nokia, NAVTEQ and TSystems and holds one patent. Actually he is Ph.D. student at the Centro de Investigaciones en Optica, A.C. His current research interests concern fiber Bragg and long period gratings, fiber lasers, and fiber laser noise.

Yuri O. Barmenkov (M'11) received his Ph.D. degree in radio-physics and electronics from the St. Petersburg State Technical University, St. Petersburg, Russia, in 1991. He was a Professor Assistant and then a Senior Lecturer with the Department of Experimental Physics of this University, from 1991 to 1996. Since 1996, he has been a Research Professor at the Centro de Investigaciones en Optica, Leon, Guanajuato, Mexico. He is National Researcher (SNI-III), Mexico, and Regular Member of the Mexican Academy of Sciences. He has co-authored over 160 scientific papers and holds 4 patents. His research activity includes fiber Bragg gratings, fiber sensors, fiber lasers, fiber laser dynamics, and nonlinear optics.

Alexander V. Kir'yanov (M'11) received his Ph.D. degree in laser physics from the A.M. Prokhorov General Physics Institute (GPI), Russian Academy of Sciences (RAS), Moscow, Russia, in 1995. He has been with the GPI since 1987. Since 1998, he has been a Research Professor at the Centro de Investigaciones en Óptica (CIO), Leon, Mexico. He is National Researcher (SNI-III), Regular Member of the Mexican Academy of Sciences, and Senior Member of the Optical Society of America. He has been co-author of over 200 scientific papers and holds 4 patents (Mexico, Russia, and USA); his current interests concern solid-state and fiber lasers and nonlinear optics of solid state and optical fiber.

Antonio A. Carrascosa received his B.S. degree in Physics in 1998 and M.D. in Photonics in 2007, both from the University of Valencia, Spain. He worked for 10 years in private sector in research companies such as F. V. Centro de Estudios Ambientales del Mediterráneo (F. V. CEAM) and ChyLa. Since 2010, he works at the Laboratory of Fiber Optics (LFO) of the University of Valencia. Currently, he is Ph.D. student at the Department of Applied Physics at this University. His current research interests concern fiber lasers, modelocked fiber lasers, broadband light sources, and fabrication of photonics crystal fibers.

Jose L. Cruz received his Ph.D. degree in physics from the University of Valencia, Valencia, Spain, in 1992. Initially, his career focused on microwave devices for radar applications; afterward, he joined the Optoelectronics Research Center, University of Southampton, Southampton, UK, where he was working in optical fiber fabrication. He is currently a Professor in the Department of Applied Physics, University of Valencia, where he is conducting research on fiber lasers and amplifiers, photonic crystal fibers, fiber gratings, microwave photonics and sensors.

Miguel V. Andrés (M'97) is Professor at the Department of Applied Physics of the University of Valencia, Spain. He received his Ph.D. degree in physics from the University of Valencia, Spain, in 1985. Since 1983, he has successively served as Assistant Professor, Lecturer, and Professor in the Department of Applied Physics, University of Valencia, Valencia, Spain. After postdoctoral stay (1984-1987) at the Department of Physics, University of Surrey, UK, he has founded the Laboratory of Fiber Optics at the University of Valencia (www.uv.es/lfo). His current research interests include photonic crystal fibers, in-fiber acousto-optics, fiber lasers and new fiberbased light sources, fiber sensors, optical microcavities, microwave photonics, and waveguide theory. His research activity includes an increasing number of collaborations with Latin American universities and research institutes of Mexico, Argentina, and Brazil, among others. From 2006 to 2016, he was member of the External Evaluation Committee of the Centro de Investigaciones en Óptica, México. In 1999, he was awarded the Premio Cooperación Universidad-Sociedad 1999 of the Universidad de Valencia. Since 2009, he is a Member (Académico Correspondiente) of the Real Academia de Ciencias de Zaragoza. 